\title{
Generalized Identities of Companion Fibonacci-Like Sequences
}

\author{
Bijendra Singh $^{1}$, Shikha Bhatnagar ${ }^{{ }^{*}}$, Omprakash Sikhwal $^{2}$ \\ ${ }^{1}$ School of Studies in Mathematics, Vikram University, Ujjain, India \\ ${ }^{2}$ Department of Mathematics, Mandsaur Institute of Technology, Mandsaur, India \\ *Corresponding author_E-mail: suhani_bhatnagar@rediffmail.com
}

\begin{abstract}
Sequences have been fascinating topic for mathematicians for centuries. Fibonacci sequence, Lucas sequence, Pell sequence, Pell-Lucas sequence, Jacobsthal sequence and Jacobsthal-Lucas sequence are most prominent examples of second order recursive sequences. In this paper, companion Fibonacci-Like sequences are introduced and presented generalized identities of companion Fibonacci-Like sequences with Jacobsthal and Jacobsthal-Lucas sequences.
\end{abstract}

Keywords: Fibonacci sequence, Lucas sequence, Companion Fibonacci-Like sequences.

\section{Introduction}

It is well known that generalized Fibonacci and Lucas numbers play an important role in many subjects such as algebra, geometry and number theory. Their various elegant properties and wide applications have been studied by many authors.

The generalization of Fibonacci sequence gives new direction for research. Fibonacci sequence and their generalization have many interesting properties and applications in almost every field of science and art [14] below [14]. Authors [1], [6], [13] have been generalized the Fibonacci sequence by preserving the recurrence relation and altering the initial conditions of the sequence, while authors [7],[10],[12] have been generalized the Fibonacci sequence by preserving the initial conditions of the sequence but altering the recurrence relation slightly. Further, authors [2],[8],[9] have been generalized Fibonacci sequence by changing recurrence relation and initial conditions both.

The Fibonacci sequence [14]is defined by the recurrence relation,

$$
F_{n}=F_{n-1}+F_{n-2}, n \geq 2 \text { with } F_{0}=0, F_{1}=1 .
$$

Lucas sequence [14] is defined by the recurrence relation,

$$
L_{n}=L_{n-1}+L_{n-2}, n \geq 2 \text { with } L_{0}=2, L_{1}=1 \text {. }
$$

The Pell sequence [4] is defined by the recurrence relation,

$$
P_{n}=2 P_{n-1}+P_{n-2}, n \geq 2 \text { with } P_{0}=0, P_{1}=1 \text {. }
$$

The companion Pell or Pell-Lucas sequence [4] is defined by the recurrence relation,

$$
Q_{n}=2 Q_{n-1}+Q_{n-2}, n \geq 2 \text { with } Q_{0}=2, Q_{1}=2 \text {. }
$$

The Jacobsthal sequence [3] is defined by the recurrence relation,

$$
J_{n}=J_{n-1}+2 J_{n-2}, n \geq 2 \text { with } J_{0}=0, J_{1}=1 \text {. }
$$

The Jacobsthal-Lucas sequence [3] is defined by the recurrence relation,

$$
j_{n}=j_{n-1}+2 j_{n-2}, n \geq 2 \text { with } j_{0}=2, j_{1}=1 \text {. }
$$

The Fibonacci- Like sequence [5] is defined by recurrence relation, 


$$
S_{n+2}=S_{n+1}+S_{n}, n \geq 0 \text { with } S_{0}=2, S_{1}=2 .
$$

First few numbers of Fibonacci-Like sequences are 2, 2, 4, 6, 10, 16, and so on.

Two companion Fibonacci-Like sequences $\left\{A_{n}\right\}$ and $\left\{C_{n}\right\}$ are introduced by authors [11]. The companion FibonacciLike sequences are not only self-related but also related to Jacobsthal and Jacobsthal-Lucas sequences.

In this paper we state and derive generalized identities of companion Fibonacci- Like sequences with Jacobsthal and Jacobsthal- Lucas sequences.

\section{Preliminaries}

Companion Fibonacci-Like sequence $\left\{A_{k}\right\}[11]$ is defined by the recurrence relation,

$$
A_{k+2}=A_{k+1}+2 A_{k} \text { for } k \geq 0 \text { with } A_{0}=2, A_{1}=2 .
$$

The first few terms of sequence $\left\{A_{k}\right\}$ are $2,2,6,10,22,42$, and so on.

Companion Fibonacci-Like sequence $\left\{C_{k}\right\}[11]$ is defined by the recurrence relation,

$$
C_{k+2}=C_{k+1}+2 C_{k} \text { for } k \geq 0 \text { with } C_{0}=2, C_{1}=0 .
$$

The first few terms of sequence $\left\{C_{k}\right\}$ are 2, 0, 4, 4, 12, 20, and so on.

The characteristic equation for both is $x^{2}-x-2=0$, which has two real roots $\mu_{1}=2$ and $\mu_{2}=-1$.

Also $\mu_{1}+\mu_{2}=1, \mu_{1}-\mu_{2}=3, \mu_{1} \mu_{2}=-2, \mu_{1}^{2}=4, \mu_{2}^{2}=1$ and $\mu_{1}-1=-\mu_{2}, \mu_{2}-1=-\mu_{1}$.

The generating function for companion Fibonacci-Like sequence $\left\{A_{k}\right\}[11]$ is given by

$$
\sum_{k=0}^{\infty} A_{k} x^{k}=\frac{2}{1-x-2 x^{2}}
$$

The generating function for companion Fibonacci-Like sequence $\left\{C_{k}\right\}$ [11] is given by

$$
\sum_{k=0}^{\infty} C_{k} x^{k}=\frac{2(1-x)}{1-x-2 x^{2}}
$$

The Binet's formula for companion Fibonacci-Like sequence $\left\{A_{k}\right\}[11]$ is given by

$$
A_{k}=2 \frac{\mu_{1}^{k+1}-\mu_{2}^{k+1}}{\mu_{1}-\mu_{2}} \text {. }
$$

The Binet's formula for companion Fibonacci-Like sequence $\left\{C_{k}\right\}[11]$ is given by

$$
C_{k}=4 \frac{\mu_{1}^{k-1}-\mu_{2}^{k-1}}{\mu_{1}-\mu_{2}}
$$

The Binet's formula for Jacobsthal sequence [3] is given by

$$
J_{k}=\frac{\mu_{1}^{k}-\mu_{2}^{k}}{\mu_{1}-\mu_{2}}
$$

The Binet's formula for Jacobsthal-Lucas sequence [3] is given by 


$$
j_{k}=\mu_{1}^{k}+\mu_{2}^{k}
$$

\section{Generalized Identities}

Now we present generalized identities of companion Fibonacci- Like sequences with Jacobsthal and Jacobsthal- Lucas sequences.

Theorem 3.1: For positive integer $k \geq 1, p \geq 0$, prove that

(i) $\quad A_{2 k+p} j_{2 k+p}=A_{4 k+2 p}+2(-2)^{2 k+p}$,

(ii) $\quad C_{2 k+p} j_{2 k+p}=C_{4 k+2 p}+2(-2)^{2 k+p}$.

Proof (i): By Binet's formula (2.5) and (2.8), we have

$$
\begin{aligned}
& A_{2 k+p} j_{2 k+p}=2\left(\frac{\mu_{1}^{2 k+p+1}-\mu_{2}^{2 k+p+1}}{\mu_{1}-\mu_{2}}\right)\left(\mu_{1}^{2 k+p}+\mu_{2}^{2 k+p}\right) \\
& =\frac{2}{\mu_{1}-\mu_{2}}\left(\mu_{1}^{4 k+2 p+1}-\mu_{2}^{4 k+2 p+1}\right)+\frac{2}{\mu_{1}-\mu_{2}}\left(\mu_{1} \mu_{2}\right)^{2 n+p}\left(\mu_{1}-\mu_{2}\right) \\
& A_{2 k+p} j_{2 k+p}=A_{4 k+2 p}+2(-2)^{2 k+p} .
\end{aligned}
$$

Proof (ii): By Binet's formula (2.6) and (2.8), we have

$$
\begin{aligned}
& C_{2 k+p} j_{2 k+p}=4\left(\frac{\mu_{1}^{2 k+p-1}-\mu_{2}^{2 k+p-1}}{\mu_{1}-\mu_{2}}\right)\left(\mu_{1}^{2 k+p}+\mu_{2}^{2 k+p}\right) \\
& =\frac{4}{\mu_{1}-\mu_{2}}\left(\mu_{1}^{4 k+2 p-1}-\mu_{2}^{4 k+2 p-1}\right)+\frac{4}{\mu_{1}-\mu_{2}}\left(\mu_{1} \mu_{2}\right)^{2 k+p}\left(\frac{1}{\mu_{1}}-\frac{1}{\mu_{2}}\right) \\
& C_{2 k+p} j_{2 k+p}=C_{4 k+2 p}+2(-2)^{2 k+p} .
\end{aligned}
$$

Corollary 3.2: For positive integer $k \geq 1, p \geq 0$, prove that

(ii)

$$
\begin{aligned}
& A_{2 k+p} j_{2 k+p}=2 J_{4 k+2 p+1}+2(-2)^{2 k+p}, \\
& C_{2 k+p} j_{2 k+p}=4 J_{4 k+2 p-1}+2(-2)^{2 k+p} .
\end{aligned}
$$

Proof (i): By Binet's formula (2.5) and (2.8), we have

$$
\begin{aligned}
& A_{2 k+p} j_{2 k+p}=2\left(\frac{\mu_{1}^{2 k+p+1}-\mu_{2}^{2 k+p+1}}{\mu_{1}-\mu_{2}}\right)\left(\mu_{1}^{2 k+p}+\mu_{2}^{2 k+p}\right) \\
& =\frac{2}{\mu_{1}-\mu_{2}}\left(\mu_{1}^{4 k+2 p+1}-\mu_{2}^{4 k+2 p+1}\right)+\frac{2}{\mu_{1}-\mu_{2}}\left(\mu_{1} \mu_{2}\right)^{2 k+p}\left(\mu_{1}-\mu_{2}\right) .
\end{aligned}
$$

From (2.7), it follows that

$$
A_{2 k+p} j_{2 k+p}=2 J_{4 k+2 p+1}+2(-2)^{2 k+p} .
$$

Proof (ii): By Binet's formula (2.6) and (2.8), it follows that

$$
\begin{aligned}
& C_{2 k+p} j_{2 k+p}=4\left(\frac{\mu_{1}^{2 k+p-1}-\mu_{2}^{2 k+p-1}}{\mu_{1}-\mu_{2}}\right)\left(\mu_{1}^{2 k+p}+\mu_{2}^{2 k+p}\right) \\
& =\frac{4}{\mu_{1}-\mu_{2}}\left(\mu_{1}^{4 k+2 p-1}-\mu_{2}^{4 k+2 p-1}\right)+\frac{4}{\mu_{1}-\mu_{2}}\left(\mu_{1} \mu_{2}\right)^{2 k+p}\left(\frac{1}{\mu_{1}}-\frac{1}{\mu_{2}}\right) .
\end{aligned}
$$

From (2.7), it follows that

$$
C_{2 k+p} j_{2 k+p}=4 J_{4 k+2 p-1}+2(-2)^{2 k+p} .
$$

Following identities can be derived same as theorem (3.1) and corollary (3.2). 
Theorem 3.3: For positive integer $k \geq 1, p \geq 0$, prove that

(i) $\quad A_{2 k-p} j_{2 k-p}=A_{4 k-2 p}+2(-2)^{2 k-p}$,

(ii) $\quad C_{2 k-p} j_{2 k-p}=C_{4 k-2 p}-4(-2)^{2 k-p-1}$.

Corollary 3.4: For positive integer $k \geq 1, p \geq 0$, prove that

$$
A_{2 k-p} j_{2 k-p}=2 J_{4 k-2 p+1}+2(-2)^{2 k-p} \text {, }
$$

(ii)

$$
C_{2 k-p} j_{2 k-p}=4 J_{4 k-2 p-1}-4(-2)^{2 k-p-1} \text {. }
$$

Theorem 3.5: For positive integer $k \geq 1, p \geq 0$, prove that

(i) $\quad A_{2 k+p} j_{2 k-p}=A_{4 k}+(-2)^{2 k-p} A_{2 p}$,

(ii) $\quad C_{2 k+p} j_{2 k-p}=C_{4 k}+(-2)^{2 k-p} C_{2 p}$.

Corollary 3.6: For positive integer $k \geq 1, p \geq 0$, prove that

$$
A_{2 k+p} j_{2 k-p}=2\left[J_{4 k+1}+(-2)^{2 k-p} J_{2 p+1}\right]
$$

(ii)

$$
C_{2 k+p} j_{2 k-p}=4\left[J_{4 k-1}+(-2)^{2 k-p} J_{2 p-1}\right] \text {. }
$$

Theorem 3.7: For positive integer $k \geq 1, p \geq 0$, prove that

(i) $\quad A_{2 k-p} j_{2 k+p}=A_{4 k}-(-2)^{2 k-p+1} A_{2 p-2}$,

(ii) $\quad C_{2 k-p} j_{2 k+p}=C_{4 k}-(-2)^{2 k-p-1} C_{2 p+2}$.

Corollary 3.8: For positive integer $k \geq 1, p \geq 0$, prove that

$$
A_{2 k-p} j_{2 k+p}=2\left[J_{4 k+1}-(-2)^{2 k-p+1} J_{2 p-1}\right],
$$

(ii)

$$
C_{2 k-p} j_{2 k+p}=4\left[J_{4 k-1}-(-2)^{2 k-p-1} J_{2 p+1}\right] \text {. }
$$

Theorem 3.9: For positive integer $k \geq 1, p \geq 0$, prove that

(i) $\quad A_{2 k} j_{2 k+p}=A_{4 k+p}-2(-2)^{2 k+1} A_{p-2}$,

(ii) $\quad C_{2 k} j_{2 k+p}=C_{4 k+p}-(-2)^{2 k-1} C_{p+2}$.

Corollary 3.10: For positive integer $k \geq 1, p \geq 0$, prove that

$$
\begin{aligned}
& A_{2 k} j_{2 k+p}=2\left[J_{4 k+p+1}-(-2)^{2 k+1} J_{p-1}\right], \\
& C_{2 k} j_{2 k+p}=4\left[J_{4 k+p-1}-(-2)^{2 k-1} J_{p+1}\right] .
\end{aligned}
$$

Theorem 3.11: For positive integer $k \geq 1, p \geq 0$, prove that

(i) $\quad A_{2 k+p} j_{2 k}=A_{4 k+p}+4^{k} A_{p}$,

(ii) $\quad C_{2 k+p} j_{2 k}=C_{4 k+p}+4^{k} C_{p}$.

Corollary 3.12: For positive integer $k \geq 1, p \geq 0$, prove that

(i) $\quad A_{2 k+p} j_{2 k}=2 J_{4 k+p+1}+2^{2 k+1} J_{p+1}$,

(ii) $\quad C_{2 k+p} j_{2 k}=4 J_{4 k+p-1}+4^{k+1} J_{p-1}$.

Theorem 3.13: For positive integer $k \geq 1, p \geq 0$, prove that

(i)

$$
A_{2 k} j_{2 k+p+1}=A_{4 k+p+1}-(-2)^{2 k+1} A_{p-1},
$$

(ii)

$$
C_{2 k} j_{2 k+p+1}=C_{4 k+p+1}-(-2)^{2 k-1} C_{p+3} \text {. }
$$


Corollary 3.14: For positive integer $k \geq 1, p \geq 0$, prove that

(i)

$$
A_{2 k} j_{2 k+p+1}=2\left[J_{4 k+p+2}-(-2)^{2 k+1} J_{p}\right] \text {, }
$$

(ii)

$$
C_{2 k} j_{2 k+p+1}=4\left[J_{4 k+p}-(-2)^{2 k-1} J_{p+2}\right] \text {. }
$$

Theorem 3.15: For positive integer $k \geq 1, p \geq 0$, prove that

(i) $\quad A_{2 k+p+1} j_{2 k}=A_{4 k+p+1}+4^{k} A_{p+1}$,

(ii) $\quad C_{2 k+p+1} j_{2 k}=C_{4 k+p+1}+4^{k} C_{p+1}$.

Corollary 3.16: For positive integer $k \geq 1, p \geq 0$, prove that

(i)

$$
A_{2 k+p+1} j_{2 k}=2\left[J_{4 k+p+2}+4^{k} J_{p+2}\right] \text {, }
$$

(ii)

$$
C_{2 k+p+1} j_{2 k}=4\left[J_{4 k+p+1}+4^{k} J_{p}\right] \text {. }
$$

Theorem 3.17 For positive integer $k \geq 1, p \geq 0$, prove that

(i)

$$
A_{2 k} j_{2 k+p-1}=A_{4 k+p-1}-(-2)^{2 k+1} A_{p-3},
$$

(ii)

$$
C_{2 k} j_{2 k+p-1}=C_{4 k+p-1}-(-2)^{2 k-1} C_{p+1} \text {. }
$$

Corollary 3.18: For positive integer $k \geq 1, p \geq 0$, prove that

$$
A_{2 k} j_{2 k+p-1}=2\left[J_{4 k+p}-(-2)^{2 k+1} J_{p-2}\right] \text {, }
$$

(ii)

$$
C_{2 k} j_{2 k+p-1}=4\left[J_{4 k+p-2}-(-2)^{2 k-1} J_{p}\right] \text {. }
$$

Theorem 3.19: For positive integer $k \geq 1, p \geq 0$, prove that

(i) $\quad A_{2 k+p-1} j_{2 k}=A_{4 k+p-1}+4^{k} A_{p-1}$,

(ii) $\quad C_{2 k+p-1} j_{2 k}=C_{4 k+p-1}+4^{k} C_{p-1}$.

Theorem 3.20: For positive integer $k \geq 1, p \geq 0$, prove that

(i)

$$
A_{2 k+p-1} j_{2 k}=2\left[J_{4 k+p}+4^{k} J_{p}\right],
$$

(ii)

$$
C_{2 k+p-1} j_{2 k}=4\left[J_{4 k+p-2}+4^{k} J_{p-2}\right] \text {. }
$$

Theorem 3.21 For positive integer $k \geq 1, p \geq 0$, prove that

(i) $\quad A_{2 k+p} j_{2 k+p+1}=A_{4 k+2 p+1}$,

(ii) $\quad C_{2 k+p} j_{2 k+p+1}=C_{4 k+2 p+1}-4(-2)^{2 k+p-1}$.

Corollary 3.22: For positive integer $k \geq 1, p \geq 0$, prove that

$$
A_{2 k+p} j_{2 k+p+1}=2 J_{4 k+2 p+2},
$$

(ii) $\quad C_{2 k+p} j_{2 k+p+1}=4 J_{4 k+2 p}-4(-2)^{2 k+p-1}$.

Theorem 3.23: For positive integer $k \geq 1, p \geq 0$, prove that

(i)

$$
A_{2 k+p+1} j_{2 k+p}=A_{4 k+2 p+1}+2(-2)^{2 k+p},
$$

(ii)

$$
C_{2 k+p+1} j_{2 k+p}=C_{4 k+2 p+1} \text {. }
$$

Corollary 3.24: For positive integer $k \geq 1, p \geq 0$, prove that

(i)

$$
A_{2 k+p+1} j_{2 k+p}=2 J_{4 k+2 p+2}+2(-2)^{2 k+p},
$$

(ii)

$$
C_{2 k+p+1} j_{2 k+p}=4 J_{4 k+2 p} \text {. }
$$


Theorem 3.25: For positive integer $k \geq 1, p \geq 0$, prove that

(i)

$$
A_{2 k+p} j_{2 k+p-1}=A_{4 k+2 p-1}+2(-2)^{2 k+p-1},
$$

(ii)

$$
C_{2 k+p} j_{2 k+p-1}=C_{4 k+2 p-1} \text {. }
$$

Corollary 3.26: For positive integer $k \geq 1, p \geq 0$, prove that

(i)

$$
A_{2 k+p} j_{2 k+p-1}=2 J_{4 k+2 p}+2(-2)^{2 k+p-1},
$$

(ii)

$$
C_{2 k+p} j_{2 k+p-1}=4 J_{4 k+2 p-2} \text {. }
$$

Theorem 3.27: For positive integer $k \geq 1, p \geq 0$, prove that

$$
A_{2 k+p-1} j_{2 k+p}=A_{4 k+2 p-1} \text {. }
$$

(ii)

$$
C_{2 k+p-1} j_{2 k+p}=C_{4 k+2 p-1}-4(-2)^{4 k+p-2} \text {. }
$$

Corollary 3.28: For positive integer $k \geq 1, p \geq 0$, prove that

$$
A_{2 k+p-1} j_{2 k+p}=2 J_{4 k+2 p},
$$

(ii)

$$
C_{2 k+p-1} j_{2 k+p}=4 J_{4 k+2 p-2}-4(-2)^{4 k+p-2} \text {. }
$$

\section{Conclusion}

In this paper we have stated and derived generalized identities of companion Fibonacci-Like sequences with Jacobsthal and Jacobsthal-Lucas sequences. Mainly Binet's formulae are used for derivation of all the identities.

\section{Acknowledgements}

The authors are thankful to the reviewers for their constructive suggestions and comments for improving the exposition of the original version.

\section{References}

[1] A. F. Horadam, the Generalized Fibonacci Sequences, the American Math. Monthly, Vol. 68, No. 5 (1961), $455-459$.

[2] A. F. Horadam, Basic Properties of a Certain Generalized Sequence of Numbers, The Fibonacci Quarterly, Vol. 3, No. 3 (1965), 161-176.

[3] A. F. Horadam, Jacobsthal Representation Numbers, the Fibonacci Quarterly, Vol. 34, No. 1 (1996), 40-54.

[4] A. F. Horadam, P. Filipponi, Real Pell and Pell-Lucas numbers with Real Subscripts, The Fibonacci Quartetly, Vol. 33, No. 5 (1995), 398406.

[5] B. Singh, O. Sikhwal, S. Bhatnagar, Fibonacci-Like Sequence and its Properties, Int. J. Contemp. Math. Sciences, Vol. 5, No. 18 (2010), 859868.

[6] D. V. Jaiswal, On a Generalized Fibonacci sequence, Labdev J. Sci. Tech. Part A, Vol. 7 (1969), 67-71.

[7] G. Y. Lee, S. G. Lee, J. S. Kim, H. K. Shin, The Binet Formula and Representations of k-Generalized Fibonacci Numbers, The Fibonacci Quarterly, Vol. 39, No. 2 (2001), 158-164.

[8] K. T. Atanassov, Remark on a New Direction for a Generalization of the Fibonacci sequence, The Fibonacci Quarterly, Vol. 33, No. 3 (1995), $249-250$.

[9] K. T. Atanassov, L. C. Atanassov, D. D. Sasselov, A New Perspective to the Generalization of the Fibonacci Sequence, The Fibonacci Quarterly, Vol. 23, No. 1 (1985), 21-28.

[10] M. Edson, O. Yayenie, A New Generalization of Fibonacci sequence and Extended Binets Formula, Integers 9 (2009), 639-654.

[11] S. Bhatnagar, B. Singh, O. Sikhwal, Some Identities of Companion Fibonacci-Like Sequences, Proceeding of International Conference on Advances in Modeling, Optimization and Computing, IIT Roorkee, India, (2011),1123-1129.

[12] S. Falcon, A. Plaza, On the Fibonacci Numbers, Chaos, Solutions \& Fractals, Vol. 32, No. 5 (2007), $1615-1624$.

[13] S. Vajda, Fibonacci \& Lucas Numbers, and the Golden Section, Theory and Applications, Ellis Horwood Ltd., Chichester, (1989).

[14] T. Koshy, Fibonacci and Lucas Numbers with Applications, Wiley-Interscience Publication, New York (2001). 